\title{
FAST FCM WITH SPATIAL NEIGHBORHOOD INFORMATION FOR BRAIN MR IMAGE SEGMENTATION
}

\author{
Abbas Biniaz ${ }^{1}$ and Ataollah Abbasi ${ }^{2}$ \\ ${ }^{1}$ M.Sc. Student, Computational Neuroscience Laboratory, Department of Biomedical Engineering, Faculty of \\ Electrical Engineering, Sahand University of Technology, Tabriz,Iran.abbass_biniaz@yahoo.com \\ ${ }^{2}$ Assistant professor, Computational Neuroscience Laboratory, Department of Biomedical Engineering, Faculty of \\ Electrical Engineering, Sahand University of Technology, Tabriz,Iran.ata.abbasi@sut.ac.ir
}

\begin{abstract}
Among different segmentation approaches Fuzzy c-Means clustering (FCM) is a welldeveloped algorithm for medical image segmentation. In emergency medical applications quick convergence of FCM is necessary. On the other hand spatial information is seldom exploited in standard FCM; therefore nuisance factors can simply affect it and cause misclassification. This paper aims to introduce a Fast FCM (FFCM) technique by incorporation of spatial neighborhood information which is exploited by a linear function on fuzzy membership. Applying proposed spatial Fast FCM (sFFCM), elapsed time is decreased and neighborhood spatial information is exploited in FFCM. Moreover, iteration numbers by proposed FFCM/sFFCM techniques are decreased efficiently. The FCM/FFCM techniques are examined on both simulated and real MR images. Furthermore, to considerably decrease of convergence time and iterations number, cluster centroids are initialized by an algorithm. Accuracy of the new approach is same as standard FCM. The quantitative assessments of presented FCM/FFCM techniques are evaluated by conventional validity functions. Experimental results demonstrate that SFFCM techniques efficiently handle noise interference and significantly decrease elapsed time.
\end{abstract}

\section{Introduction}

Medical imaging is a technique and process used to create images of the human body or parts for clinical purposes. In medical imaging, accurate detection of tissue borders is very important $[1,2]$. Image segmentation is a technique to label pixels/voxels and categorizes the image into separate sections; each section with uniformity in gray levels. Medical image segmentation extracts tissue borders in medical images. Magnetic resonance imaging (MRI) is a medical imaging modality and an important tool in the evaluation of brain diseases. Segmentation of MR images has many applications in medicine such as [1-4]:
- Identification of tissue anatomy

- Pre-and-post surgical Evaluation

- Detection of abnormal tissues such as tumors and pathological lesions

- Investigation of nervous system diseases such as MS and Alzheimer

- Evaluation of arteriosclerosis disease

- Detection of left and right ventricles

- Breast cancer detection

- Diagnosis of seizures

- Diagnosis of immune system weakness 
MR image segmentation methods are commonly based on statistical or structural properties of images [5]:

1-Methods based on statistical characteristics: the statistical features are extracted from different models and functions such as the probability distribution function of the image intensities. Statistical methods segment an image by estimation of intensity distribution and assign the class labels to their corresponding pixels. They can either be parametric or nonparametric. Both are extensively used in segmentation of brain MR images such as Markov random models (MRFs) and Bayesian network classifiers $[6,7]$.

2-Methods based on structural characteristics: in these approaches the spatial characteristics of the image like edges and regions are applied. Segmentation with structural characteristics includes several groups:

- Pixel based methods segment according to the image intensity features. Thresholding, kmeans, or fuzzy c-means clustering are pixel based approaches $[8,9]$;

- Edge based methods rely on boundary identification by tracing closed areas in the image. However they fall into the trap of false and blurred edges; also their performances are unpredictable. Edge detectors such as active contours or common edge detections (like prewitt, sobel \& canny) were plentifully used in image segmentation $[5,10]$;

- region-based methods detect similar regions based on predefined criteria. The criteria can be image intensity level, the similar tissues, image uniformity, or sharpness. Region growing is a common region based technique [11].

Commonly combination of above methods has been used for optimal segmentation of medical images. Among existing methods, FCM is a well-developed unsupervised clustering approach. Widespread applications in image segmentation caused FCM to be considered as a favorite method. Applying fuzzy membership, each pixel can be a member of all tissues with different membership degrees. FCM because of its fuzziness, has high capability to reserve more information about the orig- inal image compared to the other segmentation approaches [12, 13].

Conventional FCM doesn't have enough convergence speed in medical applications specifically in emergency circumstances. This paper presents a fast FCM algorithm based on neighborhood spatial information. Proposed FFCM technique utilizes novel rule to update cluster centers in each iteration step. The new rule significantly preserves quality of standard FCM and is faster than it. Furthermore, using dist-max algorithm [14] cluster centers are initialized before clustering process. Therefore, algorithm convergence speed increases more. Moreover proposed sFFCM algorithm by incorporation of spatial information [15] in FFCM avoids from tissue misclassification by noise interference.

The rest of this paper is as follows: In section 2-1, the conventional FCM algorithm is reviewed. Section 2-2 initializes cluster centers. Section 2-3 presents FFCM algorithm and Section 2-4 proposes $\mathrm{sFCM} / \mathrm{sFFCM}$ by incorporation of spatial information in FCM/FFCM. To assess FCM techniques, validation functions are expressed in section 3. Then in Section 4, results and discussion are presented; and section 5 involves the conclusions of this paper.

\section{Methodology}

\subsection{FCM algorithm}

The c-means families are well developed group of batch clustering type because they are "least square" models. Each cluster consists of one or more common characteristics depending on the dimension of input data. FCM developed in 1970s, assigns fuzzy memberships to each element of dataset instead of hard membership [16]. Therefore in FCM each data point belongs to multiple clusters with different membership values. Let $X=$ $\left\{x_{1}, x_{2}, \ldots, x_{n}\right\}$ denote an input vector with $n$ pixels which should be partitioned into $(2 \leq c \leq n)$ and $x_{j}$ is feature value. FCM is an iterative optimization procedure which minimizes the following cost function [15]:

$$
J_{m}(U, V)=\sum_{i=1}^{c} \sum_{j=1}^{n} u_{i j}^{m}\left\|x_{j}-v_{i}\right\|^{2}
$$


And

$$
\begin{gathered}
0 \leq u_{i j} \leq 1 \text { for } 1 \leq i \leq c, 1 \leq j \leq n, \\
0 ; \sum_{k=1}^{n} u_{i j}<n \text { for } 1 \leq i \leq c \\
\sum_{i=1}^{c} u_{i j}=1, \text { for } 1 \leq j \leq n
\end{gathered}
$$

Where $n$ is number of data points, $m$ is the fuzzy fitness grade ( $m$ equals to 1 in hard clustering and more than 1 in fuzzy clustering), $u_{i j}$ is the membership of pixel $x_{j}$ in the $i$-th cluster, $v_{i}$ is the centroid of $i$-th cluster, and $\|$.$\| is Euclidean norm.$

Since the cost function must be minimized, pixels which are close to their clusters center should have high membership values. Vice versa low membership values are assigned to pixels with data far from cluster center. In the other hand, the maximum distance between the cluster centroids leads to the optimum clustering. Membership function and cluster centers are updated by the following equations:

$$
\begin{gathered}
u_{i j} \frac{1}{\sum_{k=1}^{c}\left(\frac{\left\|x_{j}-v_{i}\right\|}{\left\|x_{j}-v_{k}\right\|}\right)^{2 /(\mathrm{m}-1)}} \\
v_{i}=\frac{\sum_{j=1}^{n} u_{i j}^{m} x_{j}}{\sum_{j=1}^{n} u_{i j}^{m}} .
\end{gathered}
$$

Starting with an initial value for each cluster center, the FCM converges to a solution for $v_{i}$ representing the local minima or a saddle point of the cost function [15]. Convergence rate can be determined by comparing the differences between the membership function or cluster centers in two successive iterations. Convergence time depends on computing time of membership functions and cluster centers in the iterations.

\subsection{Cluster center initialization}

In order to avoid the random initialization, the dist-max algorithm for the center initialization has been offered [14]. In FCM/FFCM algorithm random initialization consumes more time to algorithm be converged. Cluster center initialization algorithm for FCM/FFCM is as follows [14]:

Step1: Sorting $m_{i}$ 's in ascending order where $m_{k}=\frac{1}{p} \sum_{s=1}^{p} x_{i s}$ and $i=1,2, \ldots n$ for input vector $X=$ $\left\{x_{1}, x_{2}, \ldots, x_{n}\right\}$ which is $p$-dimensional data.
Step2: Relabeling and reorganizing the dataset matrix as $X^{\prime}=\left\{x_{1}^{\prime}, x_{2}^{\prime}, \ldots, x_{n}^{\prime}\right\}$; Partition the data in to $c$ groups and Find $n c_{k}=\lfloor n / c\rfloor$, where $n c_{k}$ is number of data points in $k$-th cluster; such that the $(j-1)$ th group contains the $(j-1)$ th $n c_{k}$ data of $X^{\prime}$, and the $c$ th group contains the remaining all elements; where $j=1,2, \ldots, c$. The number of cluster $c$ is specified according to the nature of the dataset $(1<c<n)$.

Step3: Making a distance tables that show the distance between the elements within each group. (ie) if group $k=\left\{x_{1}^{k}, x_{2}^{k}, \ldots, x_{n}^{k}\right\}$, the distance table in this group is (table 1):

Step4: Selecting the maximum distance from each distance table of groups. If $d_{i j}^{k}$ is maximum distance of $k$-th group, find the mean value $M_{k}$ of the elements $x_{i}$ and $x_{j}$ then assign centroid of $k$-th cluster as $M_{k}$, and $k=1,2, \ldots c$.

\subsection{Proposed Fast FCM}

As mentioned, high speed algorithms such as clustering ones are required in medical applications. Moreover, standard FCM doesn't have enough convergence speed especially in emergency conditions. FCM assigns $c$ membership grades to every pixel. By iteratively updating the cluster centers and membership grades for each data point, FCM moves the cluster centers to the right location within a data set. However, updating membership matrix with $c \times n$ member is a time consuming procedure. In FCM, centroids are updated by fuzzy memberships which need much time because cluster centers are selected as a fuzzy quantity. Whereas cluster centers are similar feature vectors and can be calculated by a hard membership. Hence to reduce time and amount of computations in FCM, a hard membership can be assigned to pixels for updating cluster centers in each iteration step. however, segmentation will be a fuzzy procedure. Applying hard membership, the new algorithm to update centroids is proposed as following:

Step1: For $p$-dimensional input data, rearrange $u_{i j}$ to $d_{1} \times d_{2}$ matrix; where $d_{1}$ and $d_{2}$ are input dimensions.

Step2: Set new fuzzy membership as $u_{i j}^{*}$ and label matrix as $L=\left\{L^{1}, L^{2}, \ldots L^{c}\right\}$; where $L^{k}$ is label matrix of $k$-th cluster in current iteration.

Step3: Set all data points which are correspond to 


\begin{tabular}{|c|c|c|c|c|}
\hline & $x_{1}^{k}$ & $x_{2}^{k}$ & $\cdots \cdots$ & $x_{n}^{k}$ \\
\hline$x_{1}^{k}$ & $d_{11}^{k}$ & $d_{12}^{k}$ & & $d_{1 n}^{k}$ \\
\hline$x_{2}^{k}$ & $d_{21}^{k}$ & $d_{22}^{k}$ & & $d_{2 n}^{k}$ \\
\hline$\cdot$ & $\cdot$ &. & $\ldots \ldots$ &. \\
\hline$x_{n}^{k}$ & $d_{n 1}^{k}$ & $d_{n 2}^{k}$ & $\cdots \cdots$ & $d_{n n}^{k}$ \\
\hline
\end{tabular}

Table 1. distance matrix within each data group

$L^{k}$ label matrix as $I^{k}$.

Step4: Define $I^{k}=I_{1}^{k}, I_{2}^{k}, \ldots, x_{c n_{k}}^{k}$ for $k$-th cluster, where $n c_{k}$ is number of data points in $k$-th cluster

Step5: Update centroid of $k$-th cluster by the following equation:

$$
v_{k}^{*}=\frac{\sum_{j=1}^{n c_{k}} I_{j}^{k}}{n c_{k}}
$$

\subsection{Spatial FCM/FFCM}

Neighborhood pixels have many similarities and analogous feature properties therefore they are members of the unique clusters with great probability. Nevertheless it has not been exploited in the FCM/FFCM algorithm successfully. To utilize neighborhood spatial information in image processing, the spatial function can impressively be represented as [15]:

$$
h_{i j}=\sum_{k \in N B\left(x_{j}\right)} u_{i k}
$$

The spatial function $h_{i j}$ just like the membership function $u_{i j}$, indicates the possibility that pixel $x_{j}$ belongs to $i$-th cluster. The lattice window $N B_{(} x_{j)}$ represents a $5 \times 5$ square lattice with a linear spatial function and $x_{j}$ is a pixel in center of lattice window.

If the majority of pixels in the local lattice belongs to the similar cluster, the spatial function $h_{i j}$ will denote that the pixel with great possibility is a member of the cluster [17]. Therefore incorporation of the spatial function into membership function is as follows [15]:

$$
u_{i j}^{*}=\frac{u_{i j}^{p} \times h_{i j}^{q}}{\sum_{k=1}^{c} u_{k j}^{p} \times h_{k j}^{q}} .
$$

Where $u_{i j}^{*}$ is new membership function, and the parameters $p$ and $q$ signify the comparative influence of both membership and spatial functions $u_{i j}$ and $h_{i j}$ respectively. The improved spatial FCM/FFCM with parameter $p$ and $q$ is represented as $\mathrm{sFCM}_{p, q} / \mathrm{sFFCM}_{p, q}$.

Hence new spatial fuzzy clustering is summarized in two stage process at each iteration step. In the 1st stage FCM /FFCM technique is simulated and in each iteration step membership function is updated. In the second stage spatial information of neighboring pixels are exploited in FCM /FFCM with spatial function. Therefore the new membership function in spatial domain for all pixels is updated by mapping all pixels membership functions to the spatial domain.

The proposed $\mathrm{sFCM} / \mathrm{sFFCM}$ can be summarized as follows:

Step1: Select the number of clusters $(c)$, fuzziness value $(m=2)$; initialize $V^{(0)} / V^{*(0)}$.

Step2: Update the membership matrix $\mathrm{U} / U^{*}$ by Eq.(6).

Step3: Update cluster center matrix $V / V^{*}$ for sFCM/sFFCM by Eq.(3)/ Eq.(4).

Step4: Repeat steps 3-4 until $\left\|v_{i}^{(t+1)}-v_{i}^{(t)}\right\|<\varepsilon$, where $\varepsilon$ is a small positive constant.

\section{Validation functions for fuzzy clustering}

Mostly two types of validity functions are used to evaluate the performance of clustering: fuzzy partition and geometric structure. Partition coefficient $V_{p c}$ and partition entropy $V_{p e}$ are fuzzy partition functions and defined as following $[18,19]$ : 


$$
\begin{gathered}
V_{p c}=\frac{\sum_{j=1}^{n} \sum_{i=1}^{c} u_{i j}^{2}}{n} . \\
V_{p e}=(-1) \times \frac{\sum_{j=1}^{n} \sum_{i=1}^{c} u_{i j} \log \left(u_{i j}\right)}{n} .
\end{gathered}
$$

In these equations less fuzziness shows better performance of the algorithm. As a result, the best clustering is achieved when $V_{p c}$ has maximum value (close to one) or $V_{p e}$ has minimum value (close to zero). these functions can only measure the fuzzy partition and don't have a direct access to the intensity vector. This problem can be solved using validity functions based on the geometric structure. To optimum clustering in validity functions based on the geometric structure, samples within one partition should be compacted and samples between different clusters should be separated [19]. To quantify the ratio of total variation within clusters, $V_{f s}$ and $V_{x b}$ are defined as following [18]:

$$
\begin{gathered}
V_{f s}=\sum_{j=1}^{n} \sum_{i=1}^{c} u_{i j}^{m}\left(\left\|x_{j}-v_{i}\right\|^{2}-\left\|v_{i}-\bar{v}\right\|^{2}\right) . \\
V_{x b}=\frac{\sum_{j=1}^{n} \sum_{i=1}^{c} u_{i j}^{m}\left(\left\|x_{j}-v_{i}\right\|^{2}\right)}{n *\left(\min _{i, k}\left\{\left\|x_{k}-v_{i}\right\|^{2}\right\}\right)} .
\end{gathered}
$$

Where, $v_{i} \neq v_{k}$ and minimized $V_{f s}$ or $V_{x b}$ lead to optimal clustering.

\section{Results and discussion}

To verify the effectiveness of the presented FCM techniques, they were evaluated on both synthetic and real MR images. in the simulations, images were corrupted by additive Gaussian white noise then segmented by FCM techniques. In all experiments cluster centers are initialized by dist-max algorithm.

similar results in the presence of noise show similar performances of FCM and FFCM techniques. Since T1 weighted images of the human brain use the longitudinal component of magnetic resonance imaging, they have high contrast and resolution; therefore they are proper for medical image segmentation.
Figure 1(a) is a simulated T1weighted MR image [15] which in Figure 1(b) is corrupted by additive Gaussian white noise $(\sigma=0.002)$; gray levels are 50, 100, 150, and 200. Each gray level represents a living tissue on MR image and should be specified as a separate cluster. result of clustering by FCM, FFCM, $\mathrm{sFCM}_{1,1}, \mathrm{sFFCM}_{1,1}, \mathrm{sFCM}_{0,2}$, and $\mathrm{sFFCM}_{0,2}$ techniques have been represented respectively in Figure 1 (c)-(h).

Figure 2(a) shows the simulated $\mathrm{T} 1$ weighted MR image of human brain corrupted by additive Gaussian white noise $(\sigma=0.001)$ in (b). segmentation results, including white matter (WM), gray matter (GM), and the cerebrospinal fluid (CSF) using standard FCM techniques have been represented in Figure 2 (c)-(h).

Real MR images also used to assess performance of proposed FFCM and SFFCM techniques. Experiments show that real MR images can be segmented efficiently by sFFCM method. In Figure 3 (a) real MR image acquisitioned from $3 \mathrm{~T}$ scanner is portrayed; this image is corrupted by additive Gaussian white noise $(\sigma=0.001)$ in Figure $3(b)$. segmentation results using FCM techniques have been represented in Figure 3 (c)-(h).

To do quantitative comparison between performances of all presented FCM techniques, results of different validation functions are tabulated in continue. Table 2 and 3 represents quantitative comparison of FCM, $\mathrm{sFCM}_{p, q}$, FFCM, and $\mathrm{sFFCM}_{p, q}$ approaches by the conventional fuzzy validity functions. In most cases, the validity functions based on the fuzzy partition show high similarity for the standard and fast FCM techniques. Moreover, differences between two approaches are insignificant. Most close to one in $V_{p c}$, most close to zero in $V_{p e}$ and $V_{x b}$, and greatest negative value in $V_{f s}$ demonstrate better clustering results.

In Figure 4 simulation time and iteration numbers using FCM techniques are shown. elapsed time and number of iterations are significantly decreased by the proposed FFCM and SFFCM approaches in diverse images. Moreover, in all FCM/sFFCM techniques, cluster centers were initialized by distmax algorithm. 

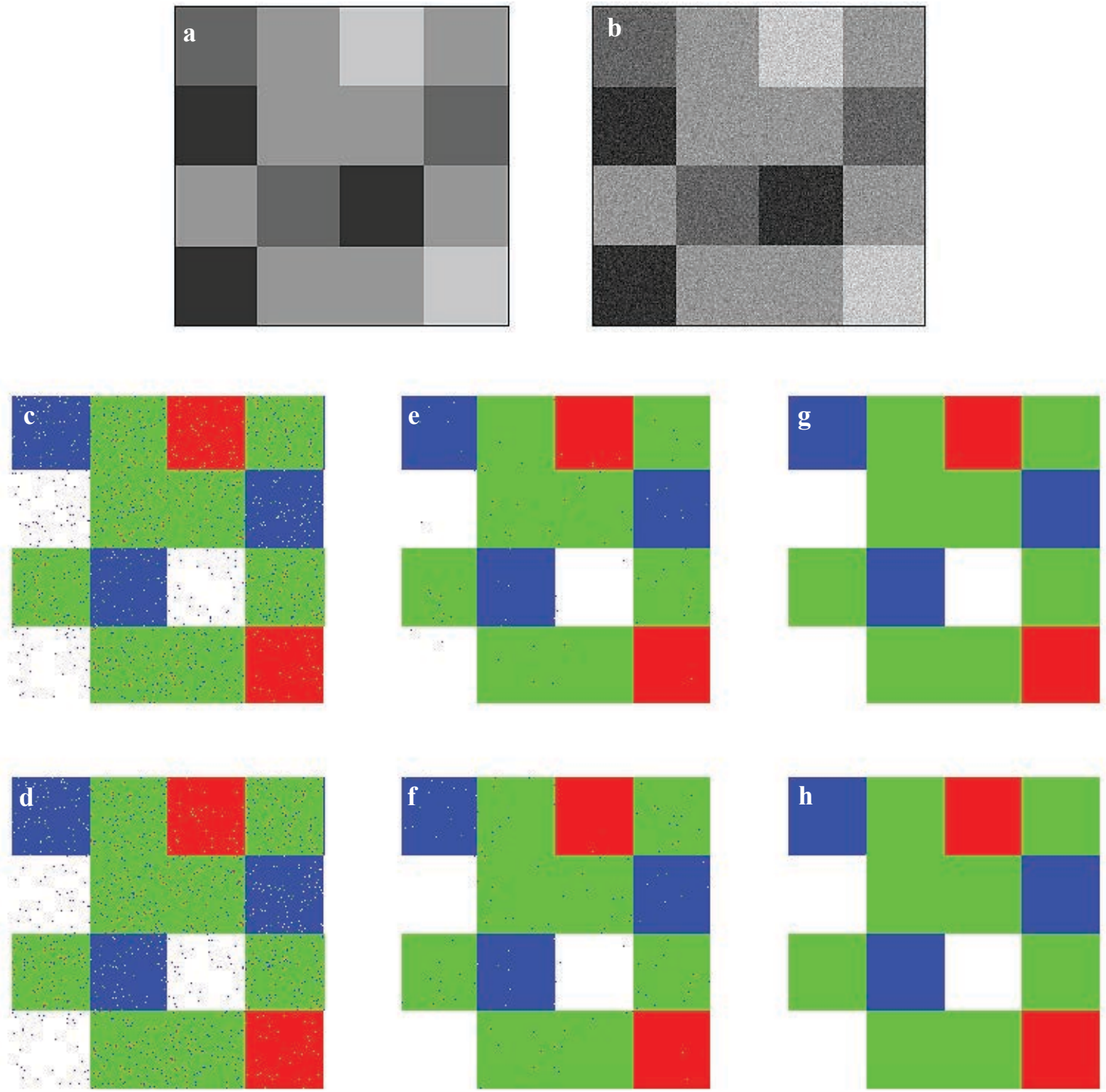

Figure 1. Segmentation results on synthetic MR image; (a) Synthetic MR image (b) corrupted by Gaussian white noise $(\sigma=0.002)$; segmented images by (c) FCM (d) FFCM, (e) $\operatorname{sFCM}_{1,1}$, (f) $\operatorname{sFCM}_{1,1}$, (g) $\mathrm{sFCM}_{0,2}$, and (h) $\mathrm{sFFCM}_{0,2}$. 

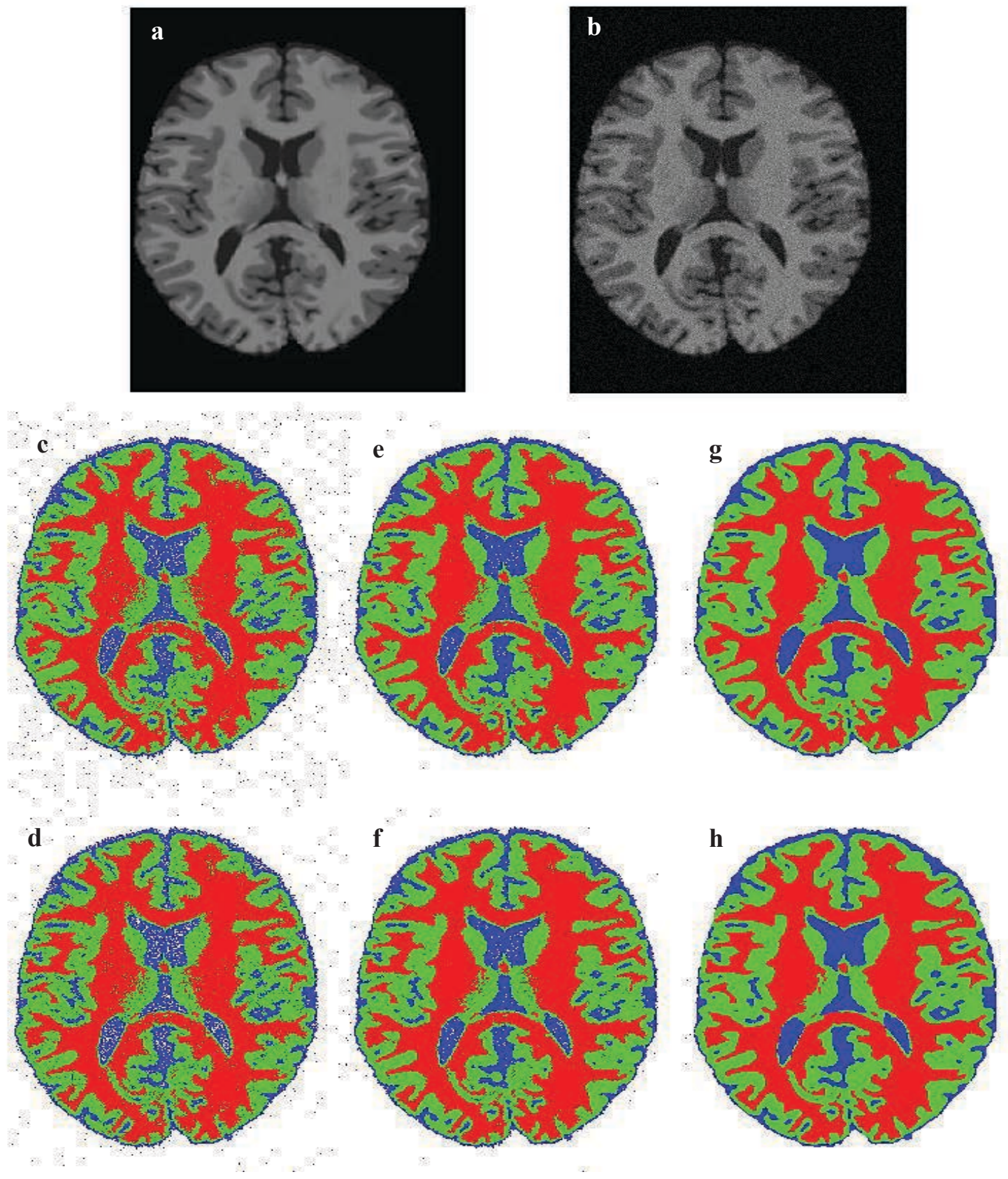

Figure 2. Segmentation results on simulated MR image; (a) simulated T1 weighted image, (b) corrupted by additive Gaussian white noise $(\sigma=0.001)$; segmented images by (c) FCM, (d) FFCM, (e)sFCM ${ }_{1,1}$, (f) $\mathrm{sFFCM}_{1,1},(\mathrm{~g}) \mathrm{sFCM}_{0,2}$, and (h) $\mathrm{sFFCM}_{0,2}$. 

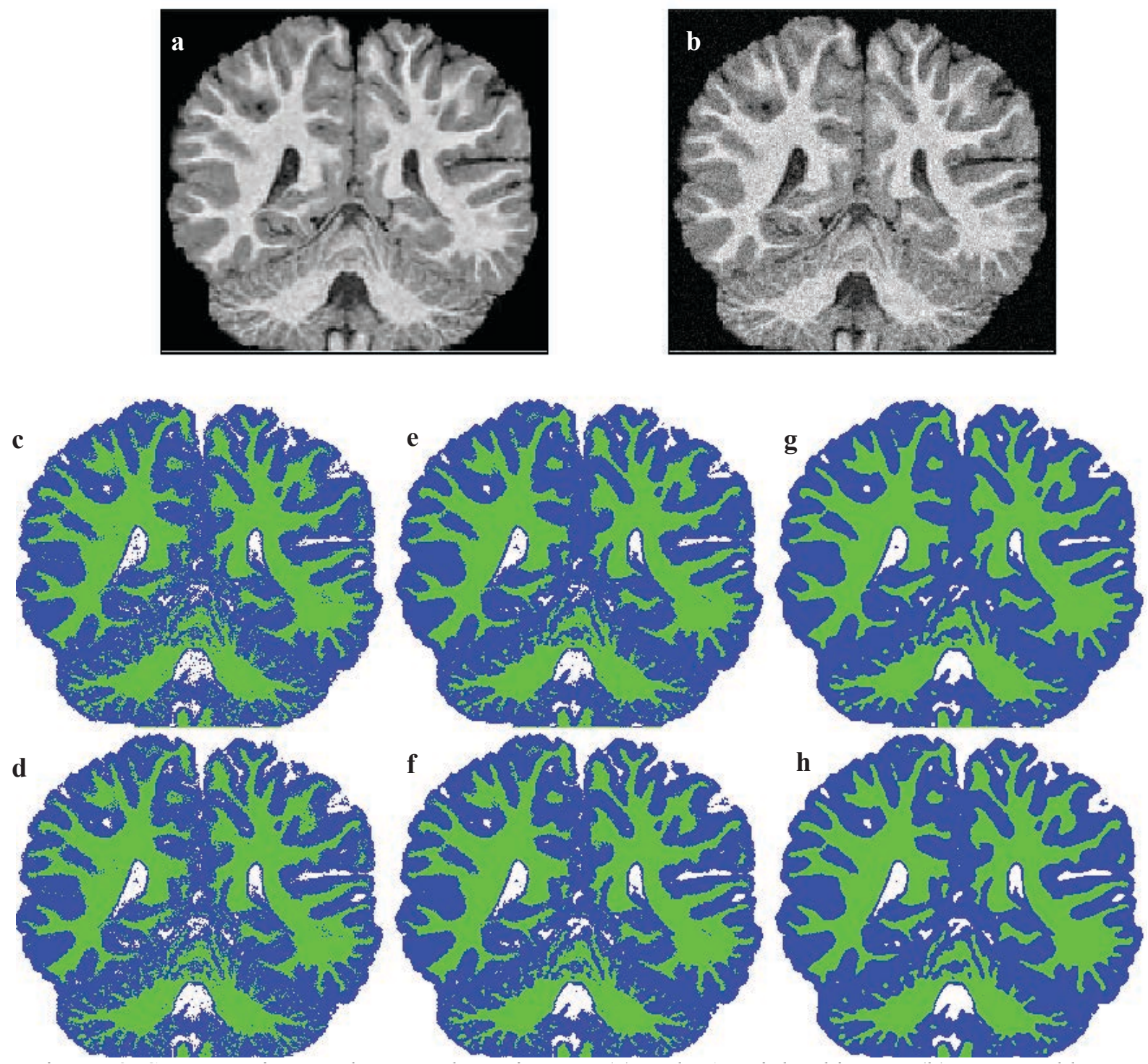

Figure 3. Segmentation results on real MR image ; (a) real T1 weighted image, (b) corrupted by additive Gaussian white noise ( $\sigma=0.001$ ); segmented images by (c) FCM (d) FFCM, (e) $\mathrm{sFCM}_{1,1}$, (f) $\mathrm{sFFCM}_{1,1}$, (g) $\mathrm{sFCM}_{0,2}$, and (h) $\mathrm{sFFCM}_{0,2}$. 
Table 2. Fuzzy partition $\left(V_{p c}\right.$ and $\left.V_{p e}\right)$ for various images using FCM/FFCM techniques

\begin{tabular}{|c|c|c|c|c|c|c|}
\hline \multirow[t]{2}{*}{ technique } & \multicolumn{2}{|c|}{ simulated 4 level MRI $(\sigma=0.002)$} & \multicolumn{2}{|c|}{ simulated MRI $(\sigma=0.001)$} & \multicolumn{2}{|c|}{ Real MRI (3T) } \\
\hline & $V_{p c}$ & $V_{p e}$ & $V_{p c}$ & $V_{p e}$ & $V_{p c}$ & $V_{p e}$ \\
\hline FCM & 0.847 & 0.135 & 0.847 & 0.131 & 0.852 & 0.117 \\
\hline FFCM & 0.847 & 0.135 & 0.848 & 0.132 & 0.842 & 0.129 \\
\hline $\mathbf{s F C M}_{1,1}$ & 0.975 & 0.024 & 0.943 & 0.044 & 0.931 & 0.051 \\
\hline sFFCM $_{1,1}$ & 0.973 & 0.025 & 0.944 & 0.043 & 0.932 & 0.051 \\
\hline $\mathbf{s F C M}_{0,2}$ & 0.969 & 0.031 & 0.916 & 0.064 & 0.907 & 0.068 \\
\hline $\mathbf{s F F C M}_{0,2}$ & 0.967 & 0.032 & 0.916 & 0.064 & 0.905 & 0.069 \\
\hline
\end{tabular}

Table 3. Geometric structure $\left(V_{x b}\right.$ and $V_{f s}$ ) for various images using FCM/FFCM techniques.

\begin{tabular}{|c|c|c|c|c|c|c|}
\hline \multirow[t]{2}{*}{ technique } & \multicolumn{2}{|c|}{$\underline{\text { simulated } 4 \text { level MRI }(\sigma=0.002)}$} & \multicolumn{2}{|c|}{ simulated MRI $(\sigma=0.001)$} & \multicolumn{2}{|c|}{ Real MRI (3T) } \\
\hline & $V_{x b}$ & $V_{f s} \times\left(-10^{6}\right)$ & $V_{x b}$ & $V_{f s} \times\left(-10^{6}\right)$ & $V_{x b}$ & $V_{f s} \times\left(-10^{6}\right)$ \\
\hline FCM & 0.038 & 145 & 0.047 & 163 & 0.065 & 306 \\
\hline FFCM & 0.038 & 143 & 0.051 & 154 & 0.078 & 248 \\
\hline $\mathrm{sFCM}_{1,1}$ & 0.046 & 160 & 0.053 & 176 & 0.069 & 320 \\
\hline $\mathbf{s F F C M}_{1,1}$ & 0.048 & 157 & 0.058 & 167 & 0.087 & 266 \\
\hline $\mathrm{sFCM}_{0,2}$ & 0.054 & 156 & 0.067 & 170 & 0.083 & 306 \\
\hline $\mathbf{s F F C M}_{0,2}$ & 0.056 & 154 & 0.070 & 163 & 0.099 & 254 \\
\hline
\end{tabular}
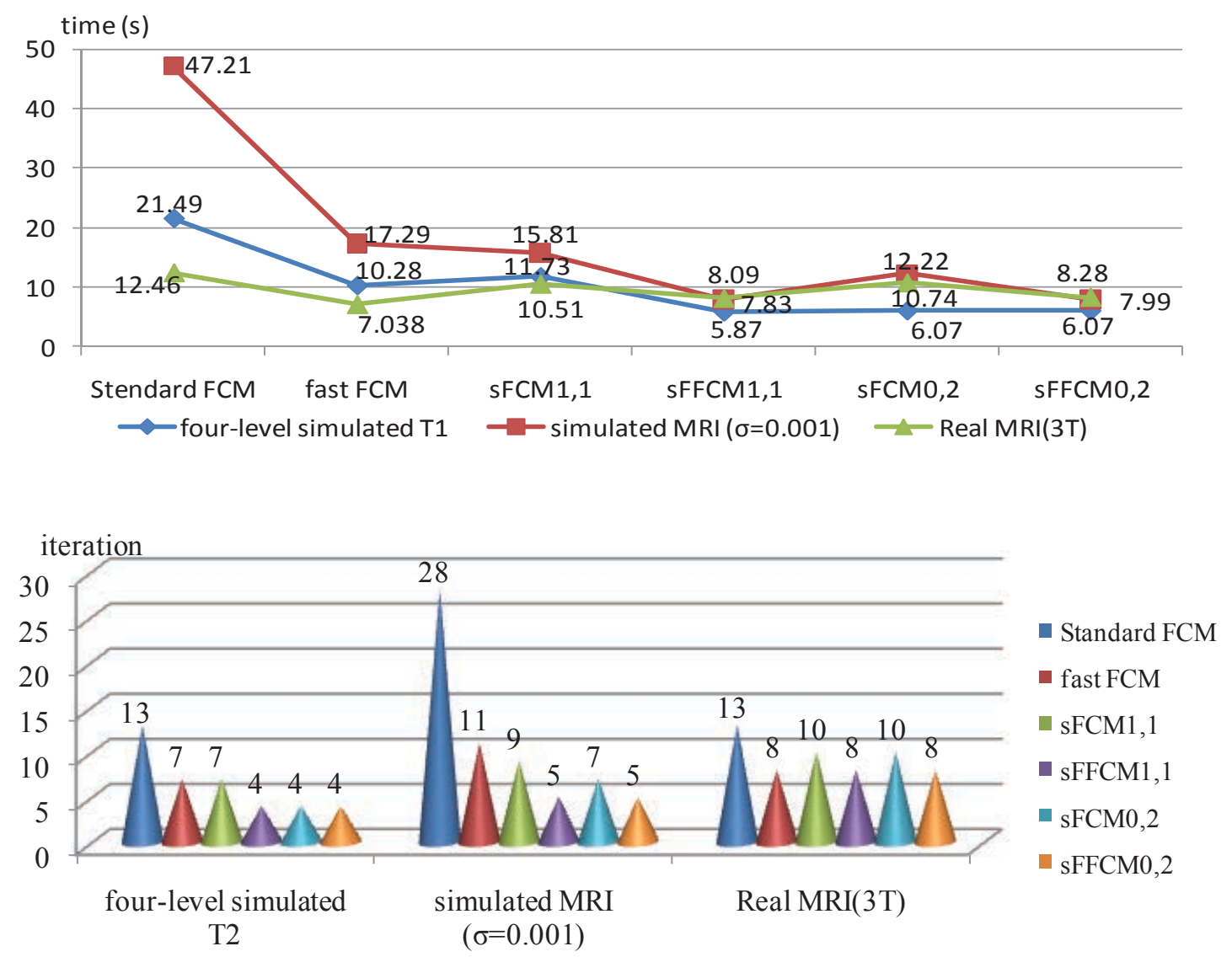

Figure 4. Figure 4: Simulation time (up) and iteration numbers (down) for FCM, FFCM, sFCM 1,1 , $\mathrm{sFFCM}_{1,1}, \mathrm{sFCM}_{0,2}$ and $\mathrm{sFFCM}_{0,2}$ on various images. 


\section{Conclusion}

In medical imaging especially in emergency situations, using high speed agents such as clustering ones are very important. standard FCM may not have required convergence rate especially in emergency conditions. Hence this paper proposed a Fast FCM technique. by new approach, time and iteration numbers relatively decreased to half of traditional FCM. Synthetic and real MR images were used in the experiments. Proposed FFCM technique decreased the computational amount and time in comparison with standard FCM. Moreover a linear spatial function was used to incorporate spatial neighborhood information in FCM/FFCM algorithm. The sFFCM algorithm decreased noise interference and handled nuisance factors effectively. Proposed sFFCM technique segmented brain MR images with the same quality of the sFCM approach whereas reduced its elapsed time. Furthermore, to more increase convergence rate and more diminish iteration numbers, cluster centers were initialized by an algorithm. Quantitative assessments of FCM/FFCM techniques were evaluated by common fuzzy validation functions. Simulation results verify efficient performance of proposed techniques.

\section{Acknowledgements}

Many thanks to the computational neuroscience laboratory (CNLab) \& ICT research center, Tabriz, Iran, to support this research. The authors also express their sincere gratitude to the anonymous referees for their helpful criticism and valuable suggestions.

\section{References}

[1] Y. Li, Z. Chi, MR Brain image segmentation based on self-organizing map network, International Journal of Information Technology, 11, 2005, 45-53.

[2] P. Dastidar, Overview of Neuroradiological MRI, International Journal of Bioelectromagnetism, 1, 1999.

[3] N. De Stefano, M.L. Bartolozzi, L. Guidi, M.L. Stromillo, A. Federico, Magnetic resonance spectroscopy as a measure of brain damage in multiple sclerosis, Journal of the Neurological Sciences, 233, 2005, 203-208.
[4] C.-W. Bong, M. Rajeswari, Multi-objective nature-inspired clustering and classification techniques for image segmentation, Applied Soft Computing, 11, 2011, 3271-3282.

[5] R.B. Dubey, M. Hanmandlu, S.K. Gupta, S.K. Gupta, The Brain MR Image Segmentation Techniques and use of Diagnostic Packages, Academic Radiology, 17, 2010, 658-671.

[6] X. Wang, H. Wang, Markov random field modeled range image segmentation, Pattern Recognition Letters, 25, 2004, 367-375.

[7] T. Celik, T. Tjahjadi, Bayesian texture classification and retrieval based on multiscale feature vector, Pattern Recognition Letters, 32, 2011, 159167.

[8] M. Siyal, L. Yu, An intelligent modified fuzzy c-means based algorithm for bias estimation and segmentation of brain MRI, Pattern Recognition Letters, 26, 2005, 2052-2062.

[9] M. Mignotte, A de-texturing and spatially constrained K-means approach for image segmentation, Pattern Recognition Letters, 32 ,2011, 359367.

[10] Q. Ge, L. Xiao, J. Zhang, Z.H. Wei, A robust patch-statistical active contour model for image segmentation, Pattern Recognition Letters, 2012.

[11] J. Fan, G. Zeng, M. Body, M.S. Hacid, Seeded region growing: an extensive and comparative study, Pattern Recognition Letters, 26, 2005, 1139-1156.

[12] A. Mekhmoukh, K. Mokrani, M. Cheriet, A modified Kernelized Fuzzy C-Means algorithm for noisy images segmentation: Application to MRI images, 2012.

[13] M. Yang, H. Tsai, A Gaussian kernel-based fuzzy c-means algorithm with a spatial bias correction, Pattern Recognition Letters, 29, 2008, 17131725.

[14] S. Ramathilagam, R. Pandiyarajan, A. Sathya, R. Devi, S.R. Kannan, Modified fuzzy c-means algorithm for segmentation of T1-T2-weighted brain MRI, Journal of Computational and Applied Mathematics, 235, 2011, 1578-1586.

[15] K. Chuang, H. Tzeng, S. Chen, J. Wu, T. Chen, Fuzzy c-means clustering with spatial information for image segmentation, Computerized Medical Imaging and Graphics, 30,2006, 9-15.

[16] J.C. Dunn, A fuzzy relative of the ISODATA process and its use in detecting compact wellseparated clusters, 1973. 
[17] Y. Li, Y. Shen, Fuzzy c-means clustering based on spatial neighborhood information for image segmentation, Systems Engineering and Electronics, Journal of, 21, 2010, 323-328.

[18] W. Wang, Y. Zhang, On fuzzy cluster validity indices, Fuzzy Sets and Systems, 158 ,2007, 20952117.
[19] K. Xiao, S.H. Ho, A. Bargiela, Automatic brain MRI segmentation scheme based on feature weighting factors selection on fuzzy c-means clustering algorithms with Gaussian smoothing, International Journal of Computational Intelligence in Bioinformatics and Systems Biology, 1, 2010, 316-331. 